\title{
Listeria innocua
}

National Cancer Institute

\section{Source}

National Cancer Institute. Listeria innocua. NCI Thesaurus. Code C86500.

A species of facultatively anaerobic, Gram positive, rod shaped bacteria in the phylum Firmicutes. This species is motile by flagella and pilli, nonsporulating and halotolerant. L. innocua is commonly found in food and in the soil, and is nonpathogenic. 